\title{
Can the host immune system promote multiple invasions of erythrocytes in vivo? Differential effects of medication and host sex in a wild malaria-like model
}

\author{
J. MARTÍnEZ-DE LA PUENTE*, S. MERINO, G. TOMÁs, J. MORENO, J. MORALES, \\ E. LOBATO and S. GARCÍA-FRAILE
}

Departamento de Ecología Evolutiva, Museo Nacional de Ciencias Naturales-C.S.I.C., C $\backslash$ Fosé Gutiérrez Abascal, 2. E-28006 Madrid, Spain

(Received 11 September 2006; revised 3 October 2006; accepted 4 October 2006; first published online 4 December 2006)

\author{
SUMMARY
}

Multiple invasions (MIs) or infections, i.e. those by more than 1 parasite in the same erythrocyte, could be the result of parasite density or, alternatively, to parasite-related factors or host-related factors. According to the last possibility, to our knowledge, only 3 laboratory studies of malaria have found an increase in the occurrence of MIs when antibodies to parasite antigens were present. Therefore, we tested the possibility that MIs were influenced by the host immune status, using as model the malaria-like parasite Haemoproteus infecting blue tits (Cyanistes caeruleus). Avian hosts infected with Haemoproteus were medicated with primaquine or injected with saline solution and the density of infection and the presence of MIs counted. Medication treatment reduced significantly the density of infection by Haemoproteus in females but not in males. For females, the presence of MIs was positively associated with both the density of infection and the immunoglobulin levels on each capture, but no association was found between the treatment and the presence of MIs. For males, the density of infection but not the immunoglobulin levels was positively associated with the presence of MIs. In addition, medicated males supported more MIs than controls. Our results represent the first line of evidence in the wild for a possible role of the host immune system promoting MIs.

Key words: multiple invasions, host immunity, Haemoproteus, Plasmodium, host-parasite interactions, malaria, sexual differences.

\section{INTRODUCTION}

Traditionally, multiple invasions (MIs) of erythrocytes in malaria and malaria-like diseases have been considered as a by-product of higher parasite densities as shown by studies on infections in both human and non-humans (Wang, 1970; Fallis and Desser, 1977; Valkiūnas, 2005 ; Martínez-de la Puente et al. 2006). However, in recent years, other host- and parasite-related factors have been also considered as determinants of the occurrence of MIs. For example, Jovani (2002) proposed that MIs composed of male and female gametocytes could be a random phenomenon that could increase the transmission success in malaria parasites. Under this assumption, a 'parasite adaptive hypothesis' of MIs was proposed by Martínez-de la Puente et al. (2006), suggesting that parasites could promote MIs to increase their probability to be paired into the vector and thus to increase their possibilities of successful transmission.

Alternatively, MIs could be induced by hostrelated factors. Studies in the laboratory have found

\footnotetext{
* Corresponding author. Tel: +34914111328. Fax: +34915645078. E-mail: jmp@mncn.csic.es
}

clear associations between host immune defences and the occurrence of MIs (Miller et al. 1984; Franzén et al. 1989; Ramasamy et al. 1999). Miller et al. (1984) found that the presence of monoclonal antibodies $(\mathrm{mAb})$ to Plasmodium knowlesi reduced the total number of infected cells and increased the number of MIs. Later, Franzén et al. (1989) found an increase in both the number of single invasions and MIs by $P$. falciparum parasites in cultures when a $\mathrm{mAb}$ to an asparagine-rich protein of $P$. falciparum was added to the medium. More recently, Ramasamy et al. (1999) in an in vitro study also found an increase in the number of MIs when a specific antibody against $P$. falciparum was present. However, contrary to Franzén et al. (1989), Ramasamy et al. (1999) found that the increase in the number of MIs was not always accompanied by a change in the total number of infections. In addition, Ramasamy et al. (1999) also found that the presence of $\mathrm{IgG}$ at concentrations of $20-200 \mu \mathrm{g} / \mathrm{ml}$ from hosts previously infected with Plasmodium significantly increased the occurrence of MIs with respect to similar concentrations of $\mathrm{IgG}$ from pre-immunized animals, suggesting that the same process could also occur in vivo (Ramasamy et al. 1999). 
According to a host adaptive hypothesis of MIs, the increase in the number of MIs by the effect of host immune system may benefit hosts if they reduce the number of infected cells and consequently the anaemia provoked by the rupture of infected erythrocytes. At the same time, due to the difficulties suffered by parasite gametocytes to reach maturity in MIs (Ahmed and Mohammed, 1978; Inselburg, 1983; Martínez-de la Puente et al. 2006), hosts could therefore affect the parasite transmission success reducing the probability of infection or re-infections in their population as well as the number of parasite reservoirs.

The aim of this study was to investigate whether the level of total immunoglobulins is involved in the occurrence of MIs in nature, as has been proposed here according to previous studies in the laboratory.

\section{MATERIALS AND METHODS}

This study was conducted during the spring of 2004 using a wild population of blue tits Cyanistes caeruleus breeding in nest-boxes in Valsaín, near Segovia, central Spain $\left(40^{\circ} 53^{\prime} \mathrm{N}, 4^{\circ} 01^{\prime} \mathrm{W}\right)$. Adult birds were captured at 2 different stages of reproduction, first when attending nestlings of 3 days of age (initial capture) and 10 days later (final capture). On each capture, a blood sample was obtained from the brachial vein. One drop of the blood sample was immediately smeared, air-dried and fixed with absolute ethanol. The rest of the blood was conserved in a cold-box to minimize protein degradation until centrifugation in the laboratory (on the same day) to separate sera and cellular fractions and, later, frozen until molecular analyses. After the initial blood sample was obtained, as a part of a previous study on MIs (Martínez-de la Puente et al. 2006), birds were assigned to either medicated or control groups. Medicated birds were injected subcutaneously with $0 \cdot 1 \mathrm{ml}$ of the anti-malarial drug primaquine diluted in saline solution (concentration $1 \mathrm{mg} \cdot \mathrm{ml}^{-1}$; Sigma, St Louis, MO, USA) and controls were injected with the same volume of saline solution. Ten days later, birds were recaptured to obtain the final blood samples that were handled as described above. However, samples differ between the previous (Martínez-de la Puente et al. 2006) and the present study because we failed to get enough blood for immunoglobulin analyses from some birds in that study but here we have included other birds that were not recaptured. Brood size was quantified at each capture.

Blood smears from both captures were stained with Giemsa $(1 / 10 \mathrm{v} / \mathrm{v})$ for $45 \mathrm{~min}$ and scanned for parasites under the microscope at $100 \times$ magnification. The density of infection by Haemoproteus majoris gametocytes was calculated as the number of infected cells per 2000 red blood cells (Godfrey et al. 1987). Smears from infected individuals were selected to determine the presence or absence of MIs for the same number of erythrocytes. Serum was employed to quantify the level of total immunoglobulins by a direct enzyme-linked immunosorbent assay (ELISA), using polyclonal rabbit anti-chicken immunoglobulins conjugated with peroxidase (Sigma, St Louis, MO, USA). Absorbances were measured using a plate spectrophotometer at $\lambda=$ $405 \mathrm{~nm}$. Details and validation of the method have been described by Martínez et al. (2003).

The density of infection at each capture was logtransformed to normalize the distribution. For each sex, the effect of the treatment was analysed using repeated-measures ANOVA including the initial and final density of Haemoproteus in the blood as dependent variables (repeated-measures) and medication treatment as factor. The rest of the statistical analyses were performed by generalized linear models (GLZ) for binomial distributions. For initial capture, analyses were performed including the presence/absence of MIs in smears from infected hosts as dependent variable and the level of total immunoglobulins and the density of the parasite as continuous variables. For the final capture, the presence/absence of MIs was included as dependent variable, the density of the parasite and the level of total immunoglobulins were included as continuous variables and medication treatment as factor.

\section{RESULTS}

Females showed a significantly reduced density of infection by Haemoproteus majoris when medicated $\left(\mathrm{F}_{1,57}=5 \cdot 81, P=0 \cdot 02\right)$. By contrast, the primaquine injection did not reduce significantly the density of infection by Haemoproteus in medicated males with respect to controls $\left(\mathrm{F}_{1,53}=0 \cdot 32, P=0 \cdot 57\right)$. In addition, the level of total immunoglobulins was significantly higher in females than males at initial capture $\left(\mathrm{F}_{1,127}=15 \cdot 01, P<0 \cdot 001\right)$. This was not the case for final samples $\left(F_{1,107}=1 \cdot 80, P=0 \cdot 18\right)$. Brood size is not significantly correlated with immunoglobulins in males or females $(P>0.07$ in all cases).

\section{Female hosts}

A total of 66 and 54 females infected by Haemoproteus were trapped on the initial and final capture respectively and their level of immunoglobulins measured (sample sizes are shown in Table 1). At initial capture, the presence of MIs was significantly and positively related to both the density of infection and the immunoglobulin level (Fig. 1A; density of infection: Wald $=11 \cdot 21, P<0 \cdot 001$; immunoglobulin level: Wald $=4 \cdot 03, P=0 \cdot 045)$. This was also true for the final capture (Fig. 1B; density of infection: Wald $=12.66, \quad P<0.001$; immunoglobulin level: Wald $=4 \cdot 70, P=0 \cdot 03)$. In females, the presence of 
Table 1. Sample sizes of Haemoproteus hosts of both sexes, with and without MIs, tramped at each capture

\begin{tabular}{lll}
\hline \hline & With MIs & Without MIs \\
\hline Females & & \\
$\quad$ Initial capture & 20 & 46 \\
$\quad$ Final capture & 15 & 39 \\
Males & & \\
$\quad$ Initial capture & 16 & 47 \\
Final capture & 13 & 42 \\
\hline \hline
\end{tabular}
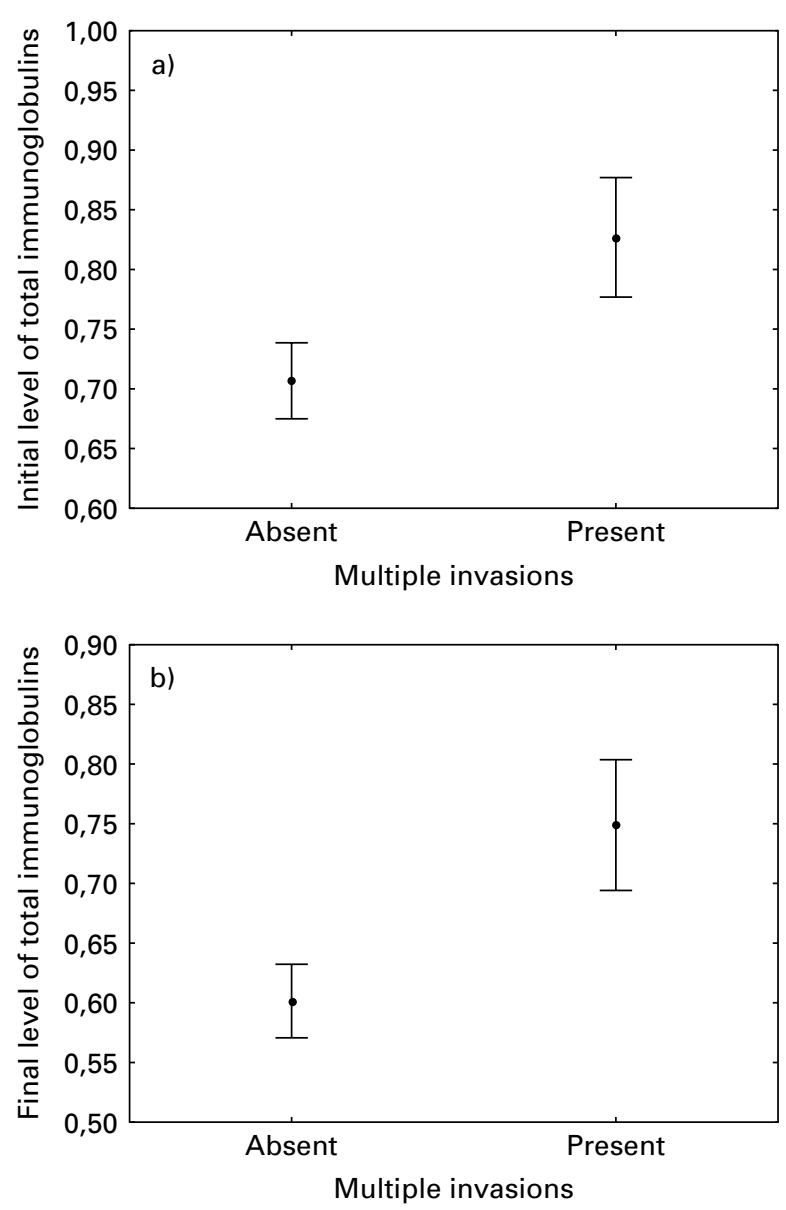

Fig. 1. Female initial (A) and final (B) immunoglobulin levels in relation to the presence of MIs. Graphs represent the associations between both variables after statistical control for the effect of the parasite density of infection (initial and final levels respectively) and the primaquine treatment (for final samples). Bars show standard error.

MIs was not associated with the primaquine treatment at final capture $($ Wald $=0 \cdot 68, P=0 \cdot 41)$.

\section{Male hosts}

A total of 63 and 55 males infected by Haemoproteus were trapped on the initial and final capture respectively and their level of immunoglobulins

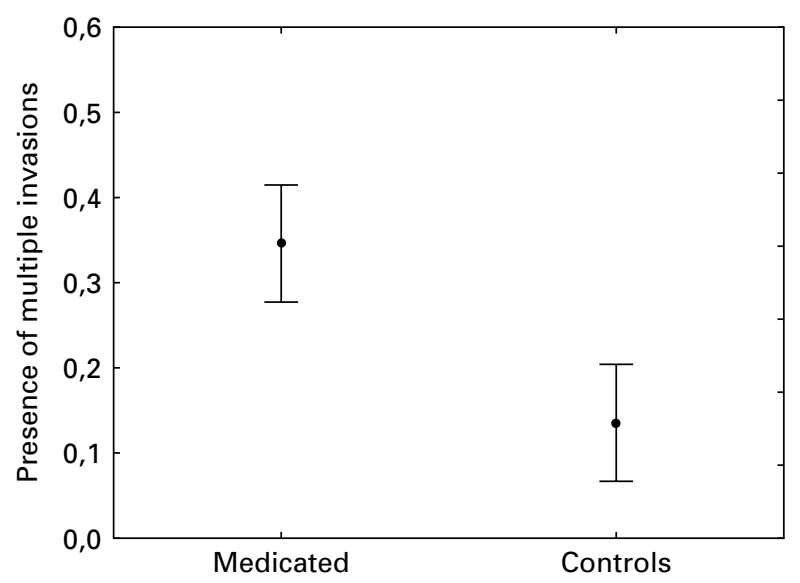

Fig. 2. Effect of primaquine injection on the occurrence of multiple invasions in host males after statistical control for the final parasite density of infection and the immunoglobulin levels. Bars show standard error.

measured (sample sizes are shown in Table 1). On the initial capture, the presence of MIs was significantly and positively related to the density of infection (Wald $=12 \cdot 04, P<0 \cdot 001)$. No association between the presence of MIs and the immunoglobulin levels was found (Wald $=0.02, P=0.89$ ). At final capture, the presence of MIs was positively associated with the density of infection in males (Wald $=11 \cdot 22$, $P<0 \cdot 001)$. No association between the presence of MIs and immunoglobulin levels was found (Wald = $0 \cdot 18, P=0 \cdot 67)$. The presence of MIs was associated with the primaquine treatment (Fig. 2 ; Wald $=4 \cdot 05$, $P=0 \cdot 044)$, medicated males presenting more MIs than controls.

There was also no significant association between brood size and parasite density or MIs $(P>0 \cdot 09$, data not shown), except for a positive correlation between final density and brood size for males once controlled for treatment effect (brood size: $\mathrm{F}_{1,53}=4 \cdot 07, P=$ $0 \cdot 049$; treatment: $\left.\mathrm{F}_{\mathbf{1 , 5 3}}=2 \cdot 24, P=0 \cdot 14\right)$. However, brood size was not significant when introduced in analyses presented in this work and the rest of the significant results were maintained, except for the relationship between medication and MIs which changed from $P=0 \cdot 044$ to $P=0 \cdot 11$ when brood size was included.

\section{DISCUSSION}

Usually, studies in human and non-human malaria and related diseases have reported that the occurrence of MIs is tightly associated with elevated parasitaemias (Wang, 1970; Fallis and Desser, 1977; Ahmed and Mohammed, 1978; Valkiūnas, 2005) independently of the host sex (Martínez-de la Puente et al. 2006, this study). However, according to the studies of Miller et al. (1984) and Ramasamy et al. (1999) the host immune system could be also involved in the occurrence of MIs. Our results in 
female blue tits clearly support this possibility, although this was not the case for males. It is known that sexual differences in behaviour, physiology and, especially, immune defence are important factors determining sexual differences in host parasite load (Zuk and McKean, 1996). In addition, the fact that males had lower immunoglobulin levels than females could be the reason, at least in part, for the absence of its effect on promoting MIs in males.

Different possibilities have been proposed to explain the mechanisms whereby antibodies promote MIs. Miller et al. (1984) proposed that MIs could be caused by a weak agglutination of merozoites by monoclonal antibodies after the rupture of infected blood cells. Later Ramasamy et al. (1999) suggested that cross-linked parasites by bivalent antibodies can recognize erythrocyte ligands and complete invasion together or following dissociation from the antibody in close proximity to the erythrocyte previous to independent invasion of the cell.

In addition, our results imply a differential effect of the primaquine treatment with respect to host sex. Several factors may account for the differential efficacy of the primaquine treatment between sexes that could affect the efficacy of the drug reducing the intensity of infection. Many other reports in medical and veterinarian literature have identified host sex as a key factor affecting drug pharmacokinetics at two different levels, the absorption and the metabolism of drugs (Gordi et al. 2002; Pinsonneault and Sadeé, 2003 ; Klein, 2004) although, in humans there are no differences in the kinetic parameters of primaquine between sexes (Elmes et al. 2006). Also, other factors tightly related to sexual characteristics, such as hormone concentrations and genetic differences, may be involved in the influence of sex on drug pharmacokinetics (Pinsonneault and Sadeé, 2003). In addition, males are slightly larger and heavier than females but were treated with the same dose of medication, which may account for the differential effect of primaquine between sexes. Although the effect of primaquine in reducing the density of infection in males could be effective for a shorter period in male versus female hosts, it could be possible that medication benefits males for a longer period if the higher number of MIs contribute to reducing the number of single infected erythrocytes. In this respect, although in females the immunoglobulin level was positively associated with the occurrence of MIs independently of the medication treatment, in males, the drug may be directly involved in the production of MIs or alternatively may induce male natural immunity other than immunoglobulin levels to promote MIs. However, the effects of medication on promoting MIs in males should be taken with caution since, when reproductive effort measured as brood size is included in analysis, the relationship between MIs and medication is no longer significant. To conclude, our results represent the first evidence for the role of the host immune system in promoting the occurrence of MIs in nature, as was expected according to a host adaptive hypothesis of MIs induction.

The study was financially supported by projects BOS200305724 to S. M. and J. M. and CGL2004-00787/BOS to J. M. Martínez-de la Puente is supported by an 'El Ventorrillo' field station grant. J. M. and E. L. are supported respectively by FPI and FPU grants from M.E.C. We thank Javier Martínez and two anonymous reviewers for enlightening discussions on the themes developed in this paper. We also thank T. Calvo and E. Sánchez for their help in the field and in the laboratory. We were authorized by J. Donés, Director of 'Centro Montes de Valsaín' to work in the study area. Dirección General del Medio Natural - Junta de Castilla y León authorized the capture and ringing of birds. This paper is a contribution from the field station 'El Ventorrillo'.

\section{REFERENCES}

Ahmed, F. E. and Mohammed, A. H. H. (1978).

Studies of growth and development of gametocytes in Haemoproteus columbae Kruse. Fournal of Protozoology 25, 174-177.

Elmes, N. J., Bennett, S. M., Abdalla, H., Carthew, T. L. and Edstein, M. D. (2006). Lack of sex effect on the pharmacokinetics of primaquine. American Fournal of Tropical Medicine and Hygiene 74, 151-152.

Fallis, A. M. and Desser, S. S. (1977). On the species of Leucocytozoon, Haemoproteus, and Hepatocystis. In Parasitic Protozoa, Vol. 3, 1st Edn. (ed. Kreir, J. P.), pp. 239-266. Academic Press, New York.

Franzén, L., Wahlin, B., Wahlgren, M., Aslund, L., Perlmann, P., Wigzell, H. and Pettersson, U. (1989). Enhancement or inhibition of Plasmodium falciparum erythrocyte reinvasion in vitro by antibodies to an asparagines rich protein. Molecular and Biochemical Parasitology 32, 201-212.

Godfrey, R. D. Jr., Fedynich, A. M. and Pence, D. B. (1987). Quantification of hematozoa in blood smears. Fournal of Wildlife Diseases 23, 558-565.

Gordi, T., Huong, D. X., Hai, T. N., Nieu, N. T. and Ashton, M. (2002). Artemisinin pharmacokinetics and efficacy in uncomplicated-malaria patients treated with two different dosage regimens. Antimicrobial Agents and Chemotherapy 46, 1026-1031.

Inselburg, J. (1983). Gametocyte formation by the progeny of single Plasmodium falciparum schizonts. Fournal of Parasitology 69, 584-591.

Jovani, R. (2002). Malaria transmission, sex ratio and erythrocytes with two gametocytes. Trends in Parasitology 18, 537-539.

Klein, S. L. (2004). Hormonal and immunological mechanisms mediating sex differences in parasite infection. Parasite Immunology 26, 247-264.

Martínez, J., Tomás, G., Merino, S., Arriero, E. and Moreno, J. (2003). Detection of serum immunoglobulins in wild birds by direct ELISA : a methodological study to validate the technique in different species using antichicken antibodies. Functional Ecology 17, 700-706.

Martínez-de la Puente, J., Merino, S., Tomás, G., Moreno, J., Morales, J. and Lobato, E. (2006). Are multiple gametocyte infections in malarial 
parasites an adaptation to ensure fertility?

Parasitology 132, 23-28.

Miller, L. H., David, P. H., Hudson, D. E., Hadley, T. J., Richards, R. L. and Aikawa, M. (1984).

Monoclonal antibodies to a $140,000 \mathrm{~m}$.W. protein on Plasmodium knowlesi merozoites inhibit their invasion of rhesus erythrocytes. Fournal of Immunology 132, 438-442.

Prasad, R. N., Prasad, H., Virk, K. J. and Sharma, V.P. (1990). Detection of multiple invasión of erythrocytes by Plasmodium vivax. Transactions of the Royal Society of Tropical Medicine and Hygiene 41, 437-438.

Pinsonneault, J. and Sadée, W. (2003).

Pharmacogenomics of multigenic diseases: sex-specific differences in disease and treatment outcome. AAPS PharmSci 5, article 29.

Ramasamy, R., Yasawardena, S., Kanagaratnam, R., Buratti, E., Baralle, F. E. and Ramasamy, M. S. (1999). Antibodies to a merozoite surface protein promote multiple invasion of red blood cells by malaria parasites. Parasite Immunology 21, 397-407.

Valkiūnas, G. (2005). Avian Malaria Parasites and other Haemosporidia. CRC Press, Boca Raton, Florida.

Wang, C. C. (1970). Multiple invasion of erythrocytes by malaria parasites. Transactions of the Royal Society of Tropical Medicine and Hygiene 64, 268-270.

Zuk, M. and McKean, K. A. (1996). Sex differences in parasite infections: patterns and processes. International Fournal for Parasitology 26, 1009-1024. 